\title{
Chipless RFID Sensor for Corrosion Characterization Based on Frequency Selective Surface and Feature Fusion
}

\author{
Adi Mahmud Jaya Marindra ${ }^{1,2}$ and Gui Yun Tian ${ }^{1}$ \\ ${ }^{1}$ School of Engineering, Newcastle University, Newcastle upon Tyne, UK \\ ${ }^{2}$ Electrical Engineering Department, Kalimantan Institute of Technology, Balikpapan, Indonesia \\ E-mail: $\underline{\text { a.m.j.marindra2@newcastle.ac.uk; g.y.tian@newcastle.ac.uk }}$
}

Received xxxxxx

Accepted for publication $\mathrm{xxxxxx}$

Published Xxxxxx

\begin{abstract}
Chipless RFID sensors attract attention to structural health monitoring (SHM) because of its advantages of being low-cost, wireless, passive, and having multiple resonances for sensing. Its application for corrosion sensing, however, receives little attention and faces challenges in terms of sensitivity and reliability. This paper proposes a chipless RFID sensor for corrosion characterization based on frequency selective surface (FSS) and feature fusion. An FSS pattern on a substrate is designed to generate three resonances within 2-6 GHz. The ability of the FSS to characterize corrosion thickness was simulated and validated in the experiments. The experimental results using dedicated corrosion undercoating samples show that the FSS based chipless RFID sensor can be used to characterize corrosion, where the three resonance frequency features provide sensitivity and consistent monotonic relations to the corrosion progression. Furthermore, feature fusion using simple sum and confidence weighted averaging (CWA) can enhance the sensitivity and reliability of the sensor. With the lowprofile and printability of the sensor, this work paves the way for smart coatings for corrosion sensing and monitoring on metallic structures.
\end{abstract}

Keywords: Chipless RFID, corrosion, frequency selective surface, fusion, metasurfaces, structural health monitoring

\section{Introduction}

Many engineering structures and components use steel as their constitutive material due to its low cost and mechanical strength. Some examples include buildings, bridges, railways, ships, oil, and gas pipelines. However, the most significant drawback of steel is its low resistance to corrosion [1]. Since engineering structures are often deployed outdoor and exposed to soil, water, chemicals, and humid atmospheres, corrosion can attack more severely than in clean environments. According to the statistical data, corrosion is one of the dominating factors for failure mechanisms and has caused numerous catastrophic accidents [2]. Without early identification of corrosion progression and stages, structures are more vulnerable to failure leading to massive economic impact and safety issues. However, corrosion rust is typically thin and composed of a mixture and multilayer anisotropic compounds. At the same time, metal loss due to corrosion also affects the surface roughness and texture of the material [3]. Additionally, structures with the corroded area are usually covered by paint or coating layer to prevent further material degradation, and thus making detection of corrosion hidden undercoating is challenging in terms of sensitivity and reliability [4]. 


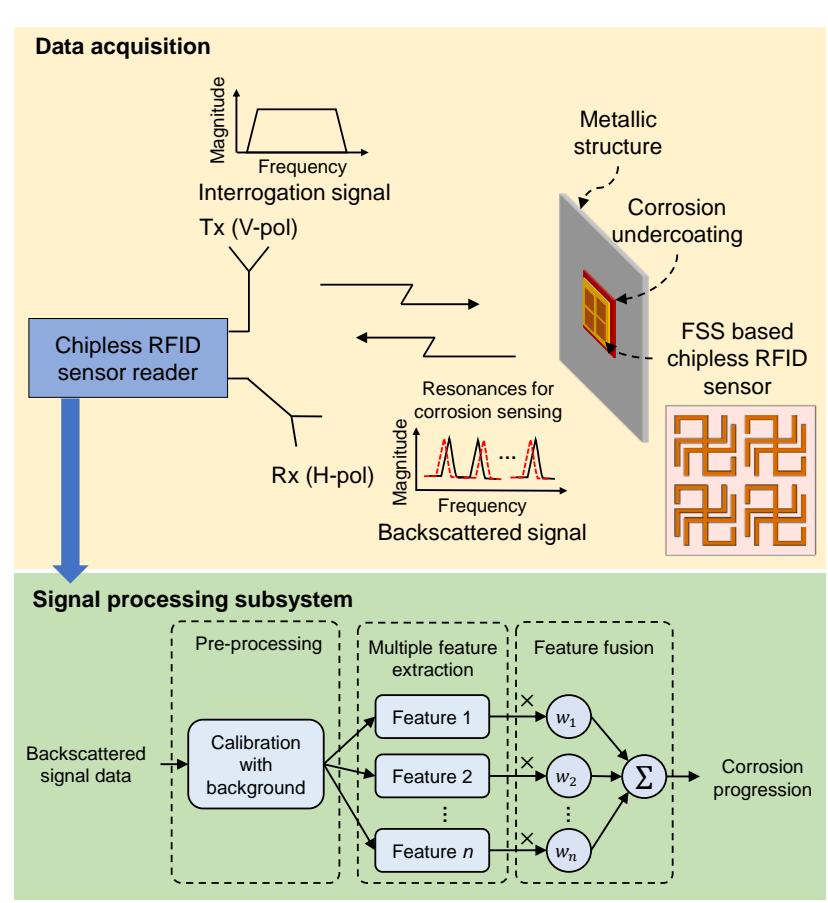

Figure 1. Operating principle of a chipless RFID sensor system for corrosion characterization using FSS based sensor and feature fusion.

The traditional non-destructive testing and evaluation (NDT\&E) techniques, such as ultrasonic testing, eddy current testing, microwave, and millimeter millimeter-wave NDT, have been used for detection and characterization of corrosion [5-7]. These techniques are generally slow, costly in time and labor, limited in range, and too cumbersome to be deployed on infrastructures. Other conventional corrosion sensors, including electrical resistance probes, electrochemical sensors, and in-line inspection tools, are recently reviewed in [8]. These active sensors require wireless sensor networks with distributed powered sources and costly for massive deployment. In recent years, therefore, radio frequency identification (RFID) antenna-based sensing is emerging and has gained interests for SHM due to the wireless, passive, and low-cost characteristics of RFID sensors [9].

Different classes of RFID sensors have been applied for corrosion detection and characterization. The low-frequency (LF) RFID [10] and high-frequency (HF) RFID sensors [11] have limited reading distance due to the inductive coupling principle. The ultra-high-frequency (UHF) RFID sensors support a relatively long reading distance, but the sensors have a large size, thick profile, and limited sensitivity $[12,13]$. Recently, chipless RFID sensors are attractive because, with the absence of chip, the sensors can be simple, printable, and durable in harsh environments. Khalifeh et al. demonstrated a retransmission-based chipless RFID sensor able to detect corroded and non-corroded microstrip stub resonators [14]. Zarifi et al. designed LC resonator and microwave ring resonator to predict potential corrosion based on water ingress on metal pipe $[15,16]$. To detect water ingress at multiple locations, Deif et al. proposed a retransmission-based flexible chipless RFID sensor using multiple spiral resonators [17]. Indeed, the multi-resonance characteristic of chipless RFID sensors is useful for detecting multiple parameters. In our previous work, a multi-resonance chipless RFID sensor can detect multiple parameters using principal component analysis (PCA), but the sensitivity and reliability are limited due to the presence of multiple influences [18]. Therefore, sensor design, sensitivity, and reliability remain challenges for chipless RFID sensors, especially for corrosion characterization.

One design approach for the implementation of chipless RFID tags and sensors is using 2D periodic conductive patterns that can be tailored to reflect EM waves with multiple resonances, namely frequency selective surface (FSS) [19,20]. FSS has been applied for designing metal-mountable chipless RFID tags using dipole patches [21], slotted aperture patches [22], and concentric square loops [23,24]. Besides, FSS has the potential for developing compact, low-profile, and highly sensitive sensors [25]. Classical FSS geometries, such as the cross, rectangular loop, and circular loop, has inspired researchers in designing functional smart materials so that strain or cracks within the material can be detected remotely [26,27]. Also, chipless dielectric constant sensors based on tilted dipole FSS and Z-shaped FSS have been reported for civil materials [28,29].

This paper introduces a chipless RFID sensor based on FSS for corrosion characterization. The contribution of this paper is two-fold. First, a novel chipless RFID sensor design based on FSS is presented with an ability to generate three resonances for corrosion sensing. Second, feature fusion is applied in this study to consolidate multiple resonance features and enhance sensitivity and reliability. Aggregation of information obtained from multiple features is useful to make robust and reliable decisions [30]. Although feature fusion is a common practice in NDT\&E and SHM [31], its application for chipless RFID sensors is rarely found. In the following sections, the principle of a chipless RFID sensor system, including the corrosion sensing approach using FSS and feature fusion using simple sum and confidence weighted averaging (CWA) are explained. Next, the design of the proposed sensor and a simulation study on corrosion sensing are presented in Section 3. Then, the experimental study and results using dedicated corrosion undercoating samples are elaborated in Section 4, followed by further discussion in Section 5. Finally, the overall conclusion of the study is given in Section 6.

\section{Principle of Chipless RFID Sensor System for Corrosion Characterization}

Sensitivity and reliability are important issues in chipless RFID sensor systems for SHM due to the complicated 


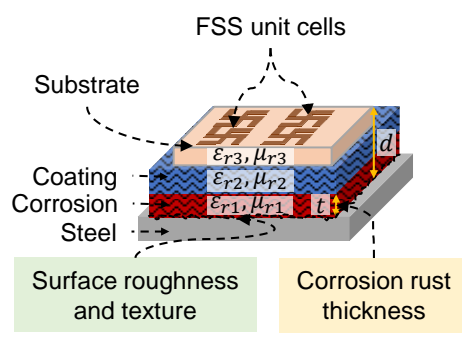

(a)

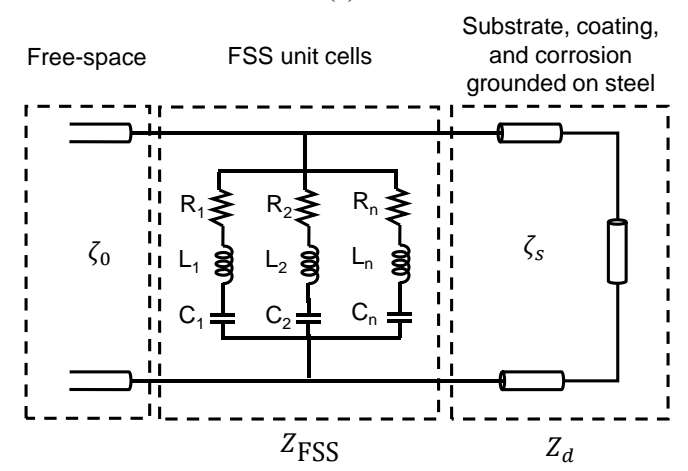

(b)

Figure 2. Corrosion sensing approach: (a) Simplified model of a corrosion undercoating with FSS based chipless RFID sensor on the top, (b) Equivalent-circuit model of FSS grounded over a corroded and coated steel.

corrosion morphology and inevitable measurement uncertainties. Therefore, the chipless RFID sensor system proposed in this work employs an FSS based sensor and feature fusion, as illustrated in Fig. 1. The system consists of an FSS based chipless RFID sensor and a reader for data acquisition and a signal processing subsystem. In its operation, the reader sends a broadband sweep-frequency signal through the transmitting antenna and, in turn, captures the backscattered signal at the receiving antenna. The reader in this work uses the cross-polarization reading technique to allow robust measurement in the multipath environment [32]. The orientations of the reader antennas are orthogonal; one is with vertical polarization, and another one is with horizontal polarization. With this principle, the receiving antenna picks up the cross-polar component of the backscattered signal to be recorded in the reader. Since the FSS based sensor has a frequency selective behavior, the FSS signature contains resonances that can be used as the corrosion indicators.

The backscattered signal data is processed in the signal processing subsystem, which includes pre-processing, feature extraction, and feature fusion. The pre-processing is a calibration to compensate for the effects of unwanted influences, such as the measurement background and mutual coupling between the $\mathrm{Tx}$ and $\mathrm{Rx}$ antennas. Then, multiple feature extraction is performed to the signature of the chipless RFID sensor, which contains multiple resonances for corrosion sensing. The multiple features are consolidated by multiplying and summing the features with certain weights. The weighted fusion results in a fused feature that determines the corrosion progression. In the following subsections, the corrosion sensing approach using FSS and the feature fusion method to obtain the weights are explained.

\subsection{Corrosion Sensing Approach Based on FSS}

Corrosion in steel is composed of oxides and hydroxides compounds. These compounds mainly consist of $\mathrm{FeOOH}$ (hydroxides), $\mathrm{Fe}_{2} \mathrm{O}_{3}$ (hematite and maghemite), and $\mathrm{Fe}_{3} \mathrm{O}_{4}$ (magnetite). According to [33,34], the dielectric constant of $\alpha$ $\mathrm{FeOOH}, \gamma-\mathrm{FeOOH}, \alpha-\mathrm{Fe}_{2} \mathrm{O}_{3}$, amorphous $\mathrm{Fe}_{2} \mathrm{O}_{3}$ and $\mathrm{Fe}_{3} \mathrm{O}_{4}$ at ambient temperature are 11, 2.6, 12, and 20, 4.5, and 20, respectively. With such an anisotropic morphology, it is hard to have an exact model of corrosion. Nevertheless, corrosion rust can be modeled as a dielectric material layer formed over a metal [35]. In the long-term exposure to the atmosphere, metals such as steel will continue to corrode over time. Corrosion progression involves two general stages, earlystage and later stage. In the early stage, corrosion thickness $(t)$ increases as corrosion progresses [36]. While only the proportions of the corrosion rust compounds increase in volume, the properties of compounds are less affected. In addition, as the corrosion progresses, the increase in rust thickness is irregular and thus affects the surface roughness and texture of the steel [37]. In the later stage, the metal loss is dominant, and the corrosion tends to spread rather than an increase in thickness.

An FSS placed over a grounded slab is a subwavelength resonant cavity with an input impedance approaching to infinite and a reflection phase crossing zero at the resonances; it is designated as a high-impedance surface (HIS) [24]. When the FSS is grounded over a corroded and coated steel as in Fig. 2(a), there are multiple dielectric slabs in between the FSS and the steel. An equivalent circuit model for the grounded FSS is drawn in Fig. 2(b). It involves the characteristic impedance of free space $\left(\zeta_{0}\right)$, the impedance of multi-resonance FSS $\left(Z_{\mathrm{FSS}}\right)$, and the impedance of the substrate $\left(\zeta_{\mathrm{s}}\right)$, coating, and corrosion grounded on steel $\left(Z_{d}\right)$. The resonances of the FSS are obtained for the frequencies at which the surface impedance $Z_{R}$ approaches or equals to the free space impedance. The surface impedance $Z_{R}$ is the parallel relation between $Z_{\mathrm{FSS}}$ and $Z_{d}$ as [24]:

$$
Z_{R}=\frac{Z_{d} Z_{\mathrm{FSS}}}{\left(Z_{d}+Z_{\mathrm{FSS}}\right)}
$$

For FSS with multiple resonances, the FSS impedance $Z_{\mathrm{FSS}}$ is represented by parallel RLC circuits depending on the number of resonances generated by the FSS elements. Meanwhile, the impedance of the grounded dielectric materials underneath the FSS elements can be expressed as [38,39]:

$$
Z_{d}=j \frac{\zeta_{0}}{\sqrt{\varepsilon_{r}^{\prime}+j \varepsilon_{r}^{\prime \prime}}} \tan \left(k_{0} \sqrt{\varepsilon_{r}^{\prime}+j \varepsilon_{r}^{\prime \prime} d}\right)
$$

where $d$ is the thickness of dielectric materials between the FSS unit cells and the steel and $k_{0}$ is the free space 


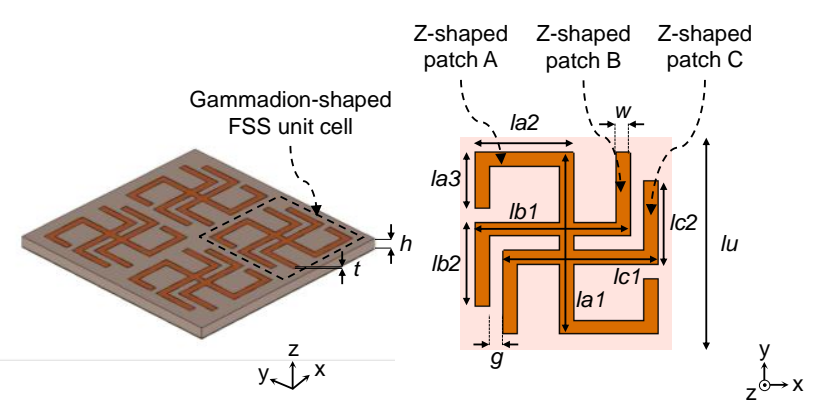

Figure 3. Geometry of the FSS based chipless RFID sensor with $2 \times 2$ unit cells.

TABLE I

DIMENSIONS OF THE FSS BASED CHIPLESS RFID SENSOR (IN MM)

\begin{tabular}{|c|c|c|c|c|c|c|c|c|c|c|c|}
\hline \hline$g$ & $h$ & $l a 1$ & $l a 2$ & $l a 3$ & $l b 1$ & $l b 2$ & $l c 1$ & $l c 2$ & $l u$ & $t$ & $w$ \\
\hline 1 & 1.52 & 13 & 7 & 4 & 11 & 6 & 11 & 6 & 15 & 0.035 & 1 \\
\hline \hline
\end{tabular}

wavenumber. Apparently, the thickness of corrosion rust is a part of $d$, and its relation is proportional to $Z_{d}$. The increase in corrosion thickness, therefore, increases the impedance $Z_{d}$ and subsequently increases $Z_{R}$. As the $Z_{R}$ increases, the resonances are shifted towards lower frequencies proportionally to the square root of the total inductance of the dielectric materials between the FSS unit cells and the steel. Hence, the corrosion progression can be reflected by the systematic change of the corrosion thickness, which affects the resonances of the prototype FSS based chipless RFID sensor.

\subsection{Feature Fusion Using Simple Sum and Confidence Weighted Averaging (CWA)}

A chipless RFID sensor with multiple resonances conveys multiple sensing information represented by features. If these features provide consistent measures of the same measurand, feature fusion can be applied to improve the sensitivity and reliability of the sensor. Assuming there are $n$ features from $n$ number of resonances, the fused feature value using simple sum rule is a non-weighted summation of all feature values $x_{i}$ :

$$
x_{\text {fused-sum }}=\sum_{i=1}^{n} x_{i}
$$

In addition to simple sum, CWA is a data fusion method for fusing multiple measurement data with respect to their variance into a more reliable estimation of the measurand. It has been used for enhancing accuracy in using multiple sensors [40]. In NDT\&E and SHM, it has been applied for mitigating noisy measurements in pulsed eddy current systems [41]. The fused feature value using CWA is calculated as the weighted average of all feature values $x_{i}$.

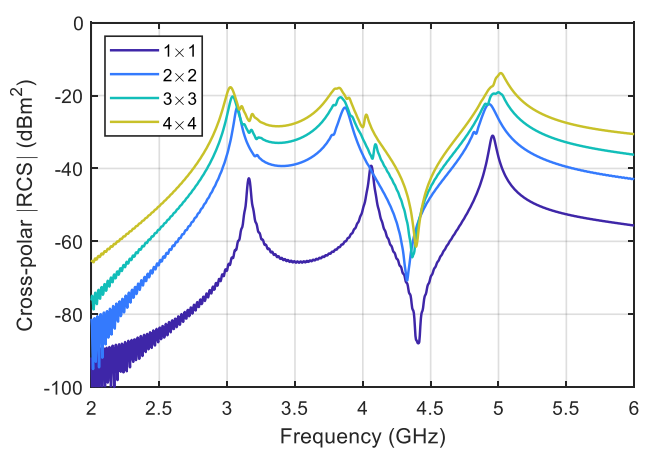

Figure 4. Simulated cross-polar RCS of the FSS based chipless RFID sensor for different number of unit cells.

$$
x_{\text {fused-CWA }}=\sum_{i=1}^{n} x_{i} w_{i}
$$

with the weighting coefficient $w_{i}$ is determined by the variance of each feature $F_{i}$ as follows

$$
w_{i}=\frac{1}{\mathbb{V}\left(F_{i}\right) \sum_{j=1}^{n} \frac{1}{\mathbb{V}\left(F_{j}\right)}}
$$

where $n$ is the number of features, $x_{i}$ represents a value obtained from the feature $F_{i}$ and $\mathbb{V}\left(F_{i}\right)$ is the approximate variance associated with the corresponding feature. The approximated variance $\mathbb{V}\left(F_{i}\right)$ is the average variances from multiple measurements and different samples. Under the assumption that the uncertainties between features are independent, the method minimizes the variance of the fused feature value. CWA finds the optimal averaging weights that minimize uncertainties in corrosion characterization.

\section{Design and Simulation of the FSS based Chipless RFID Sensor}

The FSS based chipless RFID sensor proposed in this work follows several main design criteria. First, the FSS is designed to be metal-mountable and has multiple resonances in the reflection mode, following the nature of the backscattering chipless RFID tags. The traditional FSS elements, i.e., Npoles, loop shapes, solid patch shapes, and combinations of them $[19,20]$, usually have single narrowband resonance and operated for spatial filtering applications in the transmission mode. Thus, the finite-size FSS structures that have a ground plane and act as multi-resonance absorbers are desired. Second, the FSS is designed to be depolarizing since the reader is operated in cross-polarization for robustness reading. In order to act as a wave depolarizer, the FSS unit cell geometry should be made asymmetric $[18,29,42]$. Third, the FSS is designed to be sensitive to the variation of corrosion. 


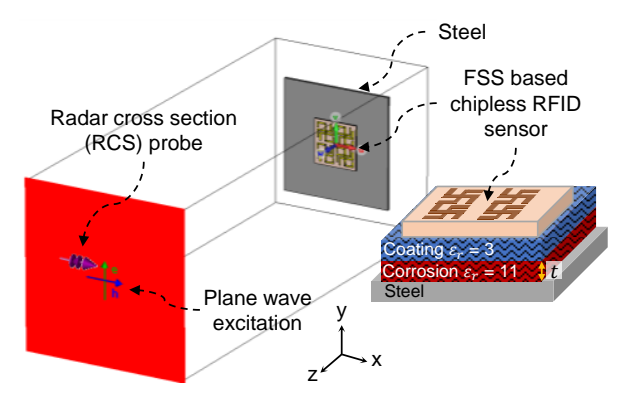

Figure 5. Simulation setup of a chipless RFID sensor system for corrosion characterization.

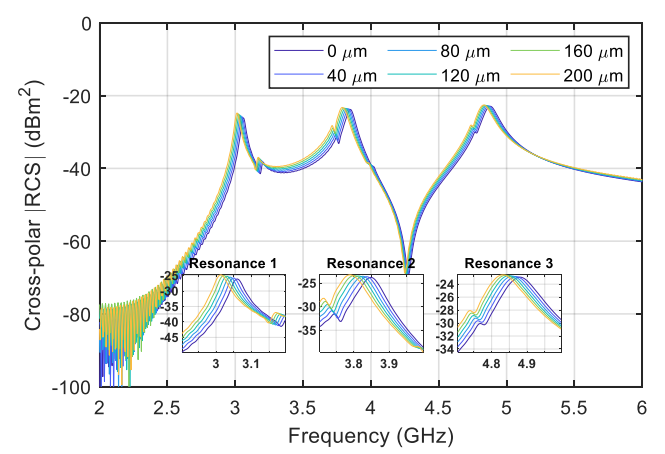

Figure 6. Simulated cross-polar RCS of the FSS based chipless RFID sensor against variations of corrosion rust thickness showing the resonance shifts to lower frequencies.

In simulation, corrosion progression can be modeled as a dielectric layer with the varied thickness between the FSS and a steel slab. The simulation of the proposed FSS against corrosion will be shown later in this section.

The geometry of the FSS based chipless RFID sensor is exhibited in Fig. 3 with the detailed dimensions listed in Table I. The sensor comprises $2 \times 2$ FSS unit cells with a gammadion shape, which is a combination of three $\mathrm{Z}$-shaped patches. The three Z-shaped patches are with different lengths and arranged asymmetrically to generate multiple resonances in a crosspolar reading. The FSS elements were designed on Rogers RO-3035 with the dielectric constant of 3.5 and tangent loss of 0.0015. The design and simulation were performed in CST microwave Studio with a steel slab placed at the backside of the FSS as a ground plane. The simulated RCS of the FSS for a different number of unit cells are shown in Fig. 4. It is apparent that the FSS generates three resonances within 2-6 $\mathrm{GHz}$ for $1 \times 1$ to $4 \times 4$ unit cell configurations. FSS allows the sensor size to be scalable so that it is conformable with the area of the monitored corrosion. A greater number of the unit cell leads to a larger periodic surface, and thus increases the RCS magnitude. The FSS unit cell has the dimensions of 15 $\mathrm{mm} \times 15 \mathrm{~mm}$, which is approximately $\lambda / 5 \times \lambda / 5$ at $4 \mathrm{GHz}$. An FSS design with $2 \times 2$ unit cells is used in this paper to suit the size of corrosion on the dedicated samples shown later in the experimental section. The overall size of the sensor is

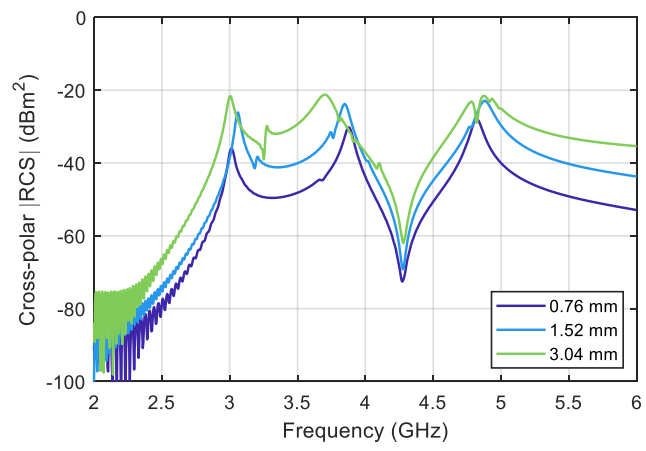

Figure 7. Simulated cross-polar RCS against the variation of the FSS substrate thickness.

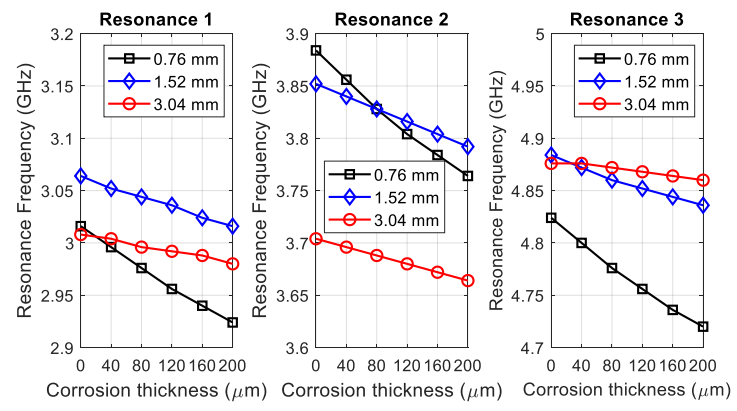

Figure 8. Simulated resonance frequencies against the variation of the FSS substrate thickness and the corrosion layer thickness.

therefore $30 \mathrm{~mm} \times 30 \mathrm{~mm} \times 1.52 \mathrm{~mm}$ and generates three resonances at $3.06,3.85$, and $4.88 \mathrm{GHz}$.

In order to observe the FSS structure as a corrosion sensor, a parametric study was performed. In the simulation setup shown in Fig. 5, two dielectric layers were present in between the FSS substrate and the metal slab. A dielectric layer below the FSS's substrate is a paint coating layer with a dielectric constant taken to be 3 [35]. Another dielectric layer between the steel and coating is corrosion rust, with the dielectric constant taken to be 11, which approximates the dielectric property of the $\alpha-\mathrm{FeOOH}$ and $\alpha-\mathrm{Fe} 2 \mathrm{O} 3$. In the parametric study, the corrosion rust thickness was varied from $0 \mu \mathrm{m}$ to $200 \mu \mathrm{m}$ with $40 \mu \mathrm{m}$ steps, while other parameters were fixed. The simulation results, as shown in Fig. 6, indicate that all three resonances were shifted to lower resonances as the corrosion thickness increased. The sensitivity of the resonances from the lowest frequency to the highest frequency is $0.22 \mathrm{MHz} / \mu \mathrm{m}, 0.30 \mathrm{MHz} / \mu \mathrm{m}$, and $0.24 \mathrm{MHz} / \mu \mathrm{m}$, respectively.

In the simulation study, the effects of the substrate thickness of the FSS to the RCS magnitude of the resonances and the sensitivity are investigated. The substrate thickness used in this work is $1.52 \mathrm{~mm}$, thus for the sake of comparison, the simulated results with the thicknesses of $0.76 \mathrm{~mm}, 1.52$ $\mathrm{mm}$, and $3.04 \mathrm{~mm}$ are presented in Fig. 7. The FSS with the $0.76-\mathrm{mm}$ substrate thickness generates resonances with low 


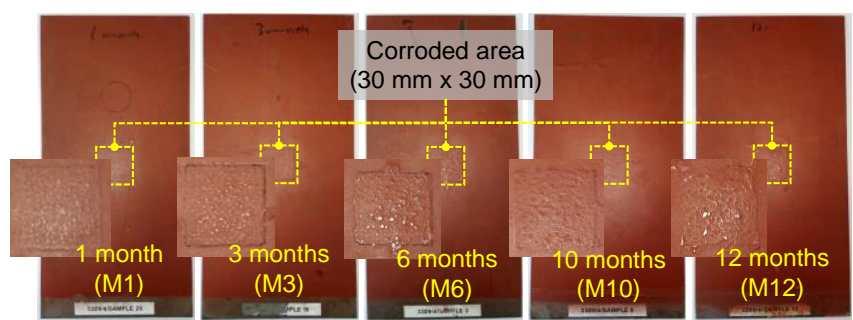

Figure 9. Dedicated corrosion undercoating samples with five different exposure period to a marine atmosphere.

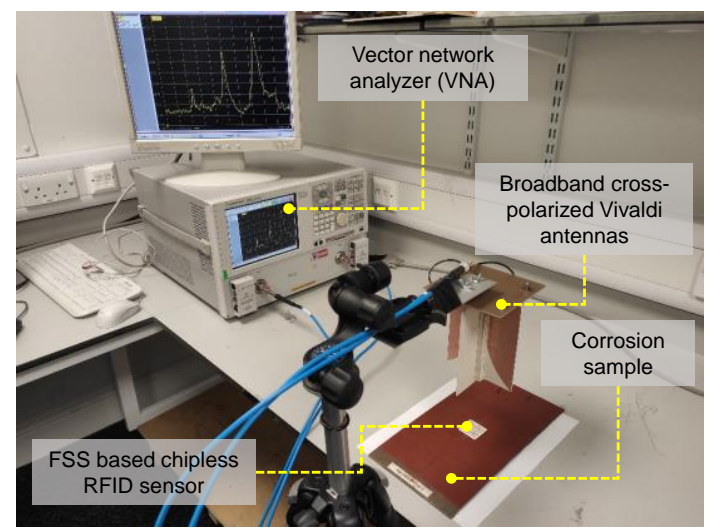

Figure 10. Experimental setup in a laboratory environment.

magnitudes of RCS around $-30 \mathrm{dBm}^{2}$, while the one with 3.04 $\mathrm{mm}$ tends to have resonances with higher magnitudes approaching $-20 \mathrm{dBm}^{2}$. In Fig. 8, the simulated resonance frequencies for different FSS substrate thickness and the corrosion thickness are presented. By seeing the steepness of the changes of resonance frequencies against the variation of corrosion thickness, the FSS with the 0.76 -mm substrate poses a higher sensitivity to the corrosion. Conversely, FSS with the 3.04-mm thick substrate tends to have lower sensitivity. Hence, the substrate thickness of $1.52 \mathrm{~mm}$ was chosen based on the consideration of having both prominent resonances and reasonable sensitivity.

\section{Experimental Study and Results}

In order to validate the simulation results and examine the FSS based chipless RFID sensor, an experimental study was carried out using dedicated corrosion undercoating samples. The corrosion undercoating samples are on mild steel S275 with the dimensions of $300 \mathrm{~mm} \times 150 \mathrm{~mm} \times 3 \mathrm{~mm}$, as depicted in Fig. 9. The center part of each sample was exposed to a marine atmosphere for different periods. Then, the entire surface of the sample was coated using nonconductive paint with a thickness of $\sim 0.1 \mathrm{~mm}$. The corroded area has dimensions of $30 \mathrm{~mm} \times 30 \mathrm{~mm}$ and different corrosion progressions according to the exposure period. The corrosion progression samples include the early-stage corrosion samples: i.e., non-corroded sample (M0), 1 month (M1), 3 months (M3), 6 months (M6); and later-stage corrosion

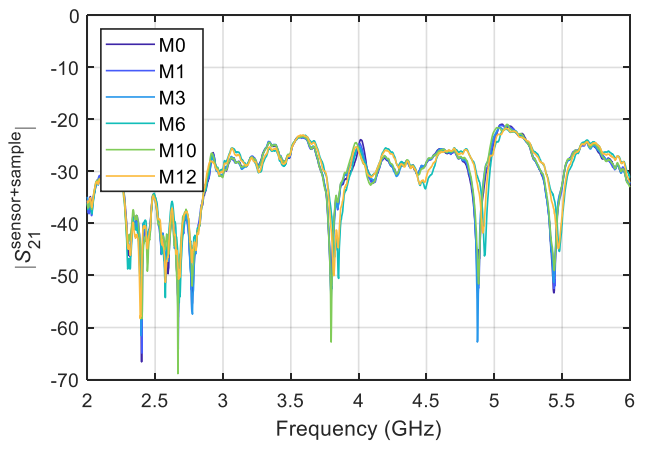

(a)

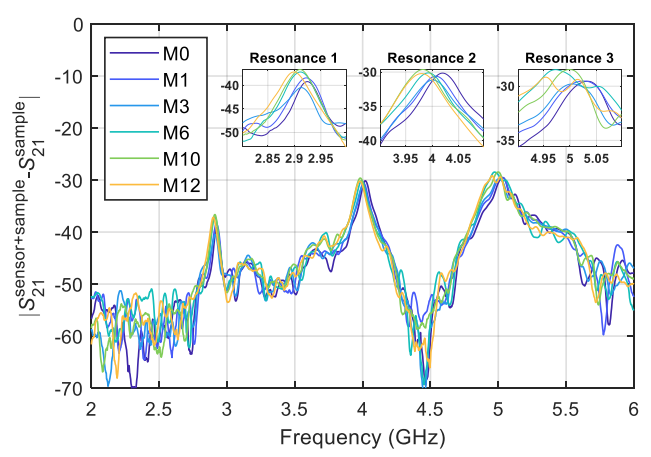

(b)

Figure 11. Measured transmission coefficient $\left(\left|S_{21}\right|\right)$ of the FSS based chipless RFID sensor for different corrosion undercoating samples: (a) Raw $\left|S_{21}\right|$ (uncalibrated). (b) Calibrated $\left|S_{21}\right|$.

samples: i.e., 10 months (M10), and 12 months (M12). For the sake of simplicity, the corrosion samples are abbreviated as M0, M1, M3, M6, M10, and M12 in the rest of this paper. The samples have been tested using different EM NDT\&E techniques $[7,10,12,13,36]$.

Fig. 10 exhibits a photograph of the experimental setup in a laboratory environment. The fabricated FSS based chipless RFID sensor was placed on the corroded area of the corrosion. Broadband cross-polarized Vivaldi antennas were employed as the reader antennas and mounted with $15 \mathrm{~cm}$ reading distance above the sensor and the corrosion sample. The design of the reader antennas is inspired by [43], which intersects two orthogonally oriented broadband Vivaldi antennas. The transmitting and receiving antennas, therefore, are compactly integrated and have the same phase center. The radiation pattern of the antennas is unidirectional, with a gain of 6 to $9 \mathrm{dBi}$ over 2-6 GHz. For measurements, the antennas were connected to the Port- 1 and Port- 2 of a VNA. Then, the transmission coefficient $\left(\left|S_{21}\right|\right)$ responses of the sensor mounted on different samples were acquired. Ten measurements were collected for each corrosion sample to consider measurement uncertainties. The $\left|S_{21}\right|$ responses of corrosion samples without the sensor were also recorded for calibration. 

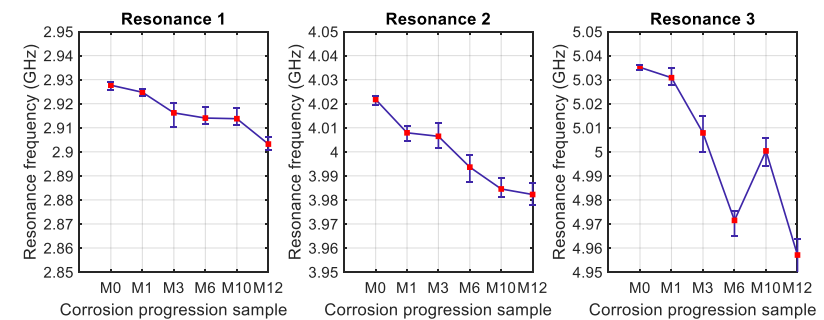

Figure 12. Characterization results of corrosion progression using individual resonance frequency features.

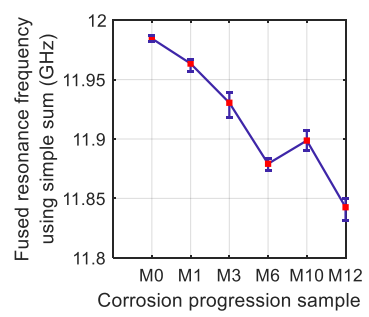

(a)

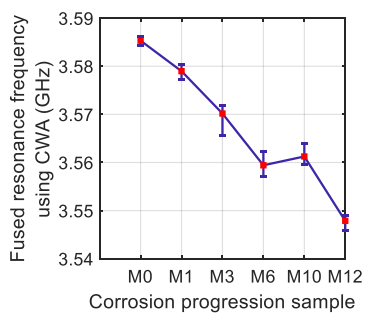

(b)
Figure 13. Characterization result of corrosion progression using the fused resonance frequency feature using: (a) Simple sum. (b) CWA.

The measured $\left|S_{21}\right|$ responses for different corrosion undercoating samples are presented in Fig. 11. The raw (uncalibrated) responses, as shown in Fig. 11(a), indicated that the resonances of the FSS based chipless RFID sensor are obscured within the polarization leakage between the Tx and $\mathrm{Rx}$ antennas. Indeed, the low echo signal from the sensor requires high isolation between the $\mathrm{Tx}$ and $\mathrm{Rx}$ antennas. To eliminate the leakage and other unwanted influences, calibration was performed by subtracting the raw $\left|S_{21}\right|$ responses with the $\left|S_{21}\right|$ responses of corrosion samples without the sensor. As shown in Fig. 11(b), the calibrated $\left|S_{21}\right|$ responses result in the signatures of the sensor, which contain three resonances at around $2.9 \mathrm{GHz}, 4 \mathrm{GHz}$, and $5 \mathrm{GHz}$. The resulting resonance frequencies are close to the simulated ones in Fig. 4 and Fig. 6, signifying a good agreement between the simulation and measurement. Furthermore, it is noticeable in Fig. 11(b) that the changes in corrosion samples lead to the variation of resonances as expected.

From the calibrated $\left|S_{21}\right|$, three resonance frequency features were extracted to characterize the corrosion progression. In Fig. 12, the values of resonance frequency features are plotted against the corrosion samples. The plotted dots indicate the average feature values while the error bars show the range of feature values, i.e., minimum and maximum values taken from ten measurements. In general, the increase of corrosion progression manifests declining trends of the resonance frequencies. The trends for three resonance frequency features are relatively consistent, but with different rates and characteristics. Resonance frequency 1, i.e., the one around $2.9 \mathrm{GHz}$, decreases less sharply, with indistinguishable corrosion progression for M6 and M10. Resonance frequency 2 decreases moderately and shows the monotonic trend of
TABLE II

SENSITIVITY AND STANDARD DEVIATION OF INDIVIDUAL RESONANCE FREQUENCY FEATURES AND THE FUSED FEATURES

\begin{tabular}{|c|c|c|}
\hline \hline Feature & Sensitivity & $\begin{array}{c}\% \text { average standard } \\
\text { deviation }\end{array}$ \\
\hline Resonance frequency 1 & $\begin{array}{c}2.3 \mathrm{MHz} / \mathrm{month} \\
0.17 \mathrm{MHz} / \mu \mathrm{m}\end{array}$ & $0.0620 \%$ \\
\hline Resonance frequency 2 & $\begin{array}{c}4.7 \mathrm{MHz} / \mathrm{month} \\
0.36 \mathrm{MHz} / \mu \mathrm{m}\end{array}$ & $0.0625 \%$ \\
\hline Resonance frequency 3 & $\begin{array}{c}10.6 \mathrm{MHz} / \mathrm{month} ; \\
0.81 \mathrm{MHz} / \mu \mathrm{m}\end{array}$ & $0.0655 \%$ \\
\hline $\begin{array}{c}\text { Fused resonance frequency } \\
\text { using simple sum }\end{array}$ & $\begin{array}{c}\mathbf{1 7 . 6} \mathrm{MHz} / \mathbf{m o n t h} ; \\
\mathbf{1 . 3 4} \mathrm{MHz} / \mu \mathrm{mm}\end{array}$ & $\mathbf{0 . 0 3 6 9 \%}$ \\
\hline $\begin{array}{c}\text { Fused resonance frequency } \\
\text { using CWA }\end{array}$ & $\begin{array}{c}4.3 \mathrm{MHz} / \mathrm{month} ; \\
0.33 \mathrm{MHz} / \mu \mathrm{m}\end{array}$ & $\mathbf{0 . 0 3 5 7 \%}$ \\
\hline \hline
\end{tabular}

corrosion progression. Meanwhile, Resonance frequency 3, which is at the highest frequency, declines most rapidly against the corrosion progression but with the M10 deviates from the overall trend. Hence, the three resonances exhibit different sensitivity and characterization results, thanks to the multi-resonance characteristic of the chipless RFID sensor so that multiple interpretations of corrosion progression can be acquired at once.

Feature fusion using simple sum and CWA were applied to find the final interpretation of the corrosion progression. By using (5), the fusion weights for the resonance frequency features were obtained as $w_{i}=[0.55 ; 0.29 ; 0.16]$. The fused resonance frequency features using simple sum and CWA are depicted in Fig. 13(a) and Fig. 13(b), respectively. The fused features using both simple sum and CWA show similar results with monotonic trends of corrosion progression for early-stage corrosions (M0 to M6). In the early stage, the value of fused resonance frequency decreases monotonically as the corrosion progression increases. However, the trend is anomalous at month 10, which is because of the abnormality of the M10 sample. According to previous studies $[7,10,12,13,36]$, the M10 and M12 samples are later-stage corrosions, in which corrosion tends to lose mass and spread rather than to increase in thickness [44]. Therefore, the characterization results, shown in Fig. 13, are reasonable and correspond to the results from the previous studies that used the same corrosion samples.

Measured using laser profilometry, the average corrosion thickness for M1, M3, M6, and M10 are $43.86 \mu \mathrm{m}, 71.72 \mu \mathrm{m}$, and $79.09 \mu \mathrm{m}$, and $70.99 \mu \mathrm{m}$, respectively [45]. The measured corrosion thickness of the M10 sample is indeed less than the M6, which validates the characterization result in this work. The sensitivity and standard deviation of individual resonance frequency features and the fused feature are calculated and summarized in Table II. The sensitivity is calculated based on the change of feature during the early stage (M0-M6) against the sample's exposure period (6 months) and against corrosion thickness at month $6(79.09 \mu \mathrm{m})$. The sensitivity against the exposure period is in $\mathrm{MHz} / \mathrm{month}$, while the one against 
TABLE III

SUMMARY OF SOME RFID SENSORS FOR CORROSION CHARACTERIZATION

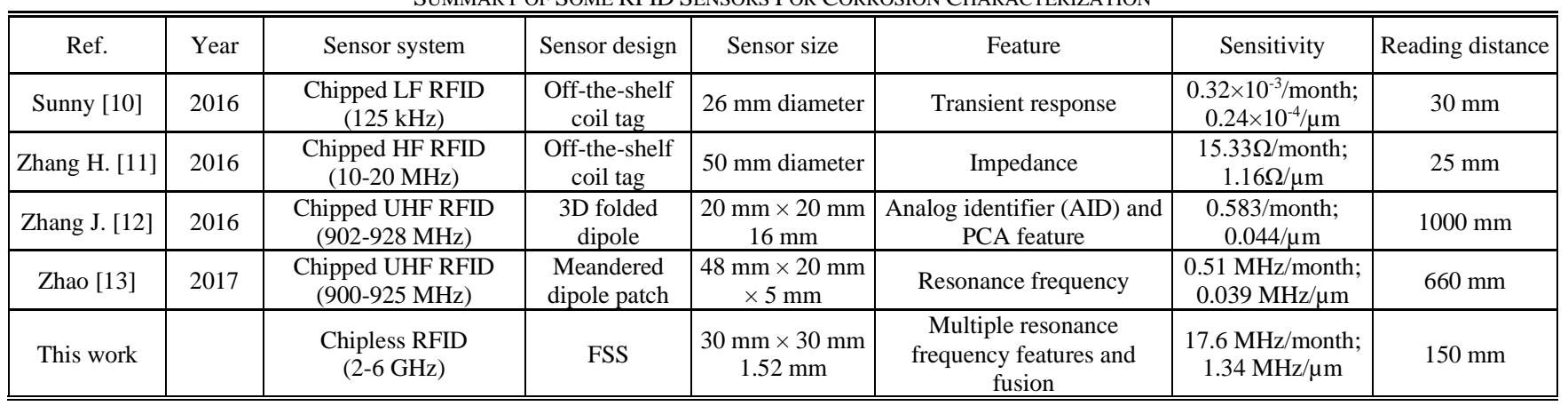

corrosion thickness is in $\mathrm{MHz} / \mu \mathrm{m}$. It can be seen that the resonances generated by the FSS based chipless RFID sensor at different frequencies provide different sensitivities to corrosion. Resonance 3 of the proposed sensor, which is at 5 $\mathrm{GHz}$, provides the highest sensitivity of $10.6 \mathrm{MHz} /$ month or $0.81 \mathrm{MHz} / \mu \mathrm{m}$. The sensitivity of the fused feature using the simple sum, which is $17.3 \mathrm{MHz} /$ month or $1.34 \mathrm{MHz} / \mu \mathrm{m}$, is equivalent to the sum of sensitivities of the individual features. Furthermore, it is revealed that the fused feature has a low average standard deviation in comparison with individual resonance frequency features. The percentage average standard deviation of the individual resonance frequency features are higher than $0.06 \%$, while feature fusion can reduce it to roughly $0.035 \%$. The low standard deviations indicate low degrees of uncertainties/errors in the measurements, thus also represents the high reliability of the sensor. In the characterization result, the high reliability is shown by the less-overlapping error bars, as in Fig. 13. Hence, the result has proven that feature fusion using simple sum and CWA can enhance the reliability of the FSS based chipless RFID sensor.

\section{Further Discussion}

In order to compare this work with other RFID sensors, a summary of RFID sensors that used the same corrosion samples is presented in Table III. Different RFID frequency bands, sensor designs, and features have been investigated for corrosion characterization. Information on sensor size, sensitivity, and reading distance is extracted for quantitative comparisons.

In terms of size, the FSS based chipless RFID sensor in this work is small and low-profile in comparison with the chipped HF and UHF RFID sensors [11-13]. Due to the lower operating frequencies and in order to work on metal, HF and UHF RFID sensors have a relatively large size and a thick profile. Regarding sensitivity, the FSS based chipless RFID sensor in this work gives a high sensitivity, especially compared to the UHF RFID sensor in [13]. The UHF RFID sensor in [13] has a sensitivity of $0.51 \mathrm{MHz} /$ month or 0.039 $\mathrm{MHz} / \mu \mathrm{m}$, while the proposed sensor has a sensitivity of 17.6 $\mathrm{MHz} / \mathrm{month}$ or $1.34 \mathrm{MHz} / \mathrm{mm}$. The high sensitivity of the chipless RFID sensor can be attributed to its operation at a higher microwave frequency range. Furthermore, it has been demonstrated that the FSS based chipless RFID sensor can characterize corrosion progression from $15 \mathrm{~cm}$ reading distance. This distance is five times longer than the capability of the LF and HF RFID sensors, but shorter than the reading distance demonstrated by UHF RFID sensors. Overall, the chipless RFID sensor in this work offers a moderate reading distance with high sensitivity and reliability compared to the RFID sensors that shared the same corrosion samples.

The above advantages in terms of size, sensitivity, and reliability come with costs of the high operating frequency range and the broad bandwidth to occupy multiple resonances. The high sensitivity in microwaves applies not only to corrosion but also to other parameters. The cross-polarization reading improves the robustness of the measurements but necessitates the reader antennas to have high isolation. Therefore, when the detection of the measurand demands high sensitivity, prior calibration with the background environment is still required to remove unwanted influences. Also, the enhancement of reliability using feature fusion needs multiple measurements to find the variances and appropriate weightings for the features. An obvious limitation with corrosion characterization using a chipless RFID sensor is in the capability of localization. Since the chipless RFID sensor can only characterize corrosion located on its backside, this technique is limited to the characterization and monitoring of localized corrosion.

Reading range, the bulkiness of reader antennas, and temperature influence are among the further practical issues. The $15-\mathrm{cm}$ reading distance demonstrated in this work may not be the best for real applications. Thus, the reading range could be improved by increasing the gain and directivity of the reader antennas, such as using reflectarray antennas [46]. Nevertheless, the bulkiness of reader antennas should also be considered in the efforts to improve the reading range. The cross-polarized Vivaldi antennas used in this work is good enough for a proof of concept in a laboratory. The compactness of reader antennas should be improved in the later stage for real applications [47]. Furthermore, in a hostile environment, temperature variations could influence the sensor's reliability. Sensor protection and temperature 
compensation, therefore, may be required in practical situations. By observing the effects of temperature variation in the signal, feature selection and fusion could be used to achieve temperature-independent corrosion monitoring [48].

\section{Conclusion}

In this paper, a chipless RFID sensor design for corrosion characterization based on FSS and feature fusion have been presented. Based on the increase of corrosion layer thickness, an FSS can be used as a battery-less, wireless, small, and lowprofile sensor to characterize corrosion progression. The FSS based chipless RFID sensor exhibits a high sensitivity to corrosion compared to the chipped RFID counterpart. Since the FSS can generate multiple resonances like chipless RFID tags, the resonance frequency features can be used as multiple predictors of corrosion progression. In this work, it has been demonstrated that the fusion of multiple resonance frequency features using simple sum and confidence weighted averaging (CWA) enhances the sensitivity and reliability of the sensor. The sensor design with feature fusion in this work can be applied to other chipless RFID sensor applications.

For future works, it would be interesting to develop the FSS-based chipless RFID sensor to operate at a higher microwave frequency range. At higher frequencies, the sensor comprises of much smaller FSS unit cells but greater in number and applied as a smart coating. This will be at the expense of fabrication complexities. Furthermore, at higher frequencies, the reader antenna can be smaller in size, has a higher radar resolution and narrower beam, allowing it to scan corrosion locations over a metallic structure.

\section{Acknowledgments}

The work of A. M. J. Marindra was supported by the Indonesia Endowment Fund for Education (LPDP) within the Ministry of Finance, Indonesia.

\section{References}

[1] Cicek V 2014 Corrosion Engineering (John Wiley \& Sons)

[2] Petrovic Z C 2016 Catastrophes caused by corrosion Vojnotehnicki glasnik/Military Technical Courier 64 1048-64

[3] Morcillo M, Fuente D de la, Diaz I and Cano H 2011 Atmospheric corrosion of mild steel Revista de Metalurgia 47 426-44

[4] Sørensen P A, Kiil S, Dam-Johansen K and Weinell C E 2009 Anticorrosive coatings: a review J Coat Technol Res 6 135-76

[5] Zhu W, Rose J L, Barshinger J N and Agarwala V S 1998 Ultrasonic Guided Wave NDT for Hidden Corrosion Detection Research in Nondestructive Evaluation 10 205-25

[6] Alamin M, Tian G Y, Andrews A and Jackson P 2012 Principal Component Analysis of Pulsed Eddy Current Response From Corrosion in Mild Steel IEEE Sensors Journal 12 2548-53

[7] Sutthaweekul R and Tian G Y 2018 Steel Corrosion Stages Characterization Using Open-Ended Rectangular Waveguide Probe IEEE Sensors Journal 18 1054-62
[8] Wright R F, Lu P, Devkota J, Lu F, Ziomek-Moroz M and Ohodnicki P R 2019 Corrosion Sensors for Structural Health Monitoring of Oil and Natural Gas Infrastructure: A Review Sensors 193964

[9] Zhang J, Tian G Y, Marindra A M J, Sunny A I and Zhao A B 2017 A Review of Passive RFID Tag Antenna-Based Sensors and Systems for Structural Health Monitoring Applications Sensors 17265

[10] Sunny A I, Tian G Y, Zhang J and Pal M 2016 Low frequency (LF) RFID sensors and selective transient feature extraction for corrosion characterisation Sensors and Actuators A: Physical 241 34-43

[11] Zhang H, Yang R, He Y, Tian G Y, Xu L and Wu R 2016 Identification and characterisation of steel corrosion using passive high frequency RFID sensors Measurement 92 421-7

[12] Zhang J and Tian G Y 2016 UHF RFID Tag Antenna-Based Sensing for Corrosion Detection amp; Characterization Using Principal Component Analysis IEEE Transactions on Antennas and Propagation 64 4405-14

[13] Zhao A, Zhang J and Tian G Y 2017 Miniaturization of UHF RFID Tag Antenna Sensors for Corrosion Characterization IEEE Sensors Journal 17 7908-16

[14] Khalifeh R, Yasri M S, Lescop B, Gallée F, Diler E, Thierry D and Rioual S 2016 Development of Wireless and Passive Corrosion Sensors for Material Degradation Monitoring in Coastal Zones and Immersed Environment IEEE Journal of Oceanic Engineering 41 776-82

[15] Zarifi M H, Sameir D and Daneshmand M 2017 Wireless passive RFID sensor for pipeline integrity monitoring Sensors and Actuators A: Physical $26124-9$

[16] Zarifi M H, Deif S, Abdolrazzaghi M, Chen B, Ramsawak D, Amyotte M, Vahabisani N, Hashisho Z, Chen W and Daneshmand M 2018 A Microwave Ring Resonator Sensor for Early Detection of Breaches in Pipeline Coatings IEEE Transactions on Industrial Electronics 65 1626-35

[17] Deif S and Daneshmand M 2019 Multi-Resonant Chipless RFID Array System for Coating Defect Detection and Corrosion Prediction IEEE Transactions on Industrial Electronics 1-1

[18] Marindra A M J and Tian G Y 2019 Multiresonance Chipless RFID Sensor Tag for Metal Defect Characterization Using Principal Component Analysis IEEE Sensors Journal 19 8037-46

[19] Panwar R and Lee J R 2017 Progress in frequency selective surface-based smart electromagnetic structures: A critical review Aerospace Science and Technology 66 216-34

[20] Anwar R S, Mao L and Ning H 2018 Frequency Selective Surfaces: A Review Applied Sciences 81689

[21] Lazaro A, Ramos A, Girbau D and Villarino R 2013 A Novel UWB RFID Tag Using Active Frequency Selective Surface IEEE Transactions on Antennas and Propagation 61 1155-65

[22] Abdulkawi W M, Sheta A-F A, Issa K and Alshebeili S A 2019 Compact Printable Inverted-M Shaped Chipless RFID Tag Using Dual-Polarized Excitation Electronics 8580

[23] Costa F, Genovesi S, Monorchio A and Manara G 2015 A Robust Differential-Amplitude Codification for Chipless RFID IEEE Microwave and Wireless Components Letters 25 832-4

[24] Costa F, Genovesi S and Monorchio A 2013 A Chipless RFID Based on Multiresonant High-Impedance Surfaces IEEE Transactions on Microwave Theory and Techniques 61 14653

[25] Song K and Mazumder P 2013 Design of Highly Selective Metamaterials for Sensing Platforms IEEE Sensors Journal 13 3377-85 
[26] Shishir M I R, Mun S, Kim H-C, Kim J W and Kim J 2017 Frequency-selective surface-based chipless passive RFID sensor for detecting damage location Structural Control and Health Monitoring 24 e2028

[27] Azemi S N, Wan Mustaffa F H, Jamlos M F, Al-Hadi A A and Soh P J 2018 Frequency Selective Surface for Structural Health Monitoring IOP Conference Series: Materials Science and Engineering 318012033

[28] Lázaro A, Villarino R, Costa F, Genovesi S, Gentile A Buoncristiani L and Girbau D 2018 Chipless Dielectric Constant Sensor for Structural Health Testing IEEE Sensors Journal 18 5576-85

[29] Costa F, Gentile A, Genovesi S, Buoncristiani L, Lazaro A, Villarino R and Girbau D 2018 A Depolarizing Chipless RF Label for Dielectric Permittivity Sensing IEEE Microwave and Wireless Components Letters 28 371-3

[30] Wu R-T and Jahanshahi M R 2020 Data fusion approaches for structural health monitoring and system identification: Past, present, and future Structural Health Monitoring 19 552-86

[31] Kralovec C and Schagerl M 2020 Review of Structural Health Monitoring Methods Regarding a Multi-Sensor Approach for Damage Assessment of Metal and Composite Structures Sensors 20826

[32] Costa F, Genovesi S and Monorchio A 2014 Chipless RFIDs for Metallic Objects by Using Cross Polarization Encoding IEEE Transactions on Antennas and Propagation 62 4402-7

[33] Chen D, Yen M, Lin P, Groff S, Lampo R, McInerney M and Ryan J 2014 A Corrosion Sensor for Monitoring the EarlyStage Environmental Corrosion of A36 Carbon Steel Materials (Basel) 7 5746-60

[34] Haynes W M 2014 CRC Handbook of Chemistry and Physics (CRC Press)

[35] Qaddoumi N N, Saleh W M and Abou-Khousa M 2007 Innovative Near-Field Microwave Nondestructive Testing of Corroded Metallic Structures Utilizing Open-Ended Rectangular Waveguide Probes IEEE Transactions on Instrumentation and Measurement 56 1961-6

[36] He Y, Tian G, Zhang H, Alamin M, Simm A and Jackson P 2012 Steel Corrosion Characterization Using Pulsed Eddy Current Systems IEEE Sensors Journal 12 2113-20

[37] Bassach P, Quintana G, Ferrer I and Ciurana J 2012 Studying the relation between corrosion and surface roughness AIP Conference Proceedings 1431 319-27

[38] Costa F and Monorchio A 2012 Closed-Form Analysis of Reflection Losses in Microstrip Reflectarray Antennas IEEE Transactions on Antennas and Propagation $604650-60$

[39] Costa F, Genovesi S, Monorchio A and Manara G 2013 A Circuit-Based Model for the Interpretation of Perfect Metamaterial Absorbers IEEE Transactions on Antennas and Propagation 61 1201-9

[40] Elmenreich W 2007 Fusion of Continuous-valued Sensor Measurements using Confidence-weighted Averaging Journal of Vibration and Control 13 1303-12

[41] Yang G, Tian G Y, Que P W and Li Y 2007 Data fusion algorithm for pulsed eddy current detection IET Science, Measurement Technology $1312-6$

[42] Vena A, Perret E and Tedjni S 2013 A Depolarizing Chipless RFID Tag for Robust Detection and Its FCC Compliant UWB Reading System IEEE Transactions on Microwave Theory and Techniques 61 2982-94

[43] Sonkki M, Sánchez-Escuderos D, Hovinen V, Salonen E T and Ferrando-Bataller M 2015 Wideband Dual-Polarized Cross-Shaped Vivaldi Antenna IEEE Transactions on Antennas and Propagation 63 2813-9
[44] Zhang H, He Y, Gao B, Tian G Y, Xu L and Wu R 2016 Evaluation of Atmospheric Corrosion on Coated Steel Using K-Band Sweep Frequency Microwave Imaging IEEE Sensors Journal 16 3025-33

[45] Zhang H 2014 Radio Frequency Non-destructive Testing and Evaluation of Defects under Insulation Ph.D. thesis (Newcastle upon Tyne, UK: Newcastle University)

[46] Khaliel M, El-Awamry A, Fawky A and Kaiser T 2018 Long reading range for the frequency coded Chipless RFID system based on reflectarray antennas International Journal of Microwave and Wireless Technologies 10 187-95

[47] Islam M A and Karmakar N C 2015 Compact Printable Chipless RFID Systems IEEE Transactions on Microwave Theory and Techniques 63 3785-93

[48] Sunny A I, Zhang J, Tian G Y, Tang C, Rafique W, Zhao A and Fan M 2019 Temperature Independent Defect Monitoring Using Passive Wireless RFID Sensing System IEEE Sensors Journal 19 1525-32 